\title{
Design and Spectral Properties of a New Displacement Senor Based on an FBG Sensor and SMA Actuators
}

\author{
Ji Yun Lu ${ }^{1 a^{*}}$, Kan Bian ${ }^{2 b}$ and Da Kai Liang ${ }^{2 C}$ \\ ${ }^{1}$ College of Civil Aviation, Nanjing University of Aeronautics \& Astronautics, Nanjing, Jiangsu \\ 210016, China \\ ${ }^{2}$ College of Aerospace Engineering, Nanjing University of Aeronautics \& Astronautics, Nanjing, \\ Jiangsu 210016, China \\ alujiyun@nuaa.edu.cn, bbk@nuaa.edu.cn, ${ }^{c}$ liangdk@nuaa.edu.cn
}

Keywords: Displacement sensor; Shape memory alloy; Fiber Bragg grating; Spectrum analysis.

\begin{abstract}
As an important factor in-service Structure health monitoring (SHM), predictions of displacement become the basis for determining the decrease of modern aircraft and aerospace structural performance. Optical fiber Bragg grating (FBG) sensors are considered an important tool for SHM , however, FBG sensors are rarely directly applied to monitor the displacement because of the intrinsic fragility. To overcome such a problem, we propose a novel method for design and curving an FBG sensor using auxiliary flexible driving function of the shape memory alloy (SMA) to monitor the displacement. The FBG sensor is curved slowly to an S shape along with SMA wire. Strain gradient of SMA which is applied by displacement loading is obtained by finite element analysis. By analyzing the spectral simulations, bandwidth of spectrum is increased and the central wavelength is changed. Spectral properties of curved FBG sensor are analyzed by stretch experiments, the results shown that its bandwidth, light intensity of peak and central wavelength are increased and the central wavelength change is linear with displacement. A new sensor based on an FBG sensor and SMA Actuators can be used to monitor displacement with displacement sensitivity of $141 \mathrm{pm} / \mathrm{mm}$.
\end{abstract}

\section{Introduction}

There is growing need for requirements of modern aircraft and aerospace structures[1]. The development of in-service Structure health monitoring (SHM) is very necessary to rapidly assess the health condition and durability of structures[2]. The strain and displacement monitoring of modern aircraft and aerospace structures play key roles in SHM fields. Compared with conventional sensors, Optical fiber Bragg grating (FBG) sensors are considered an important tool for SHM because they possess unique advantages such as flexibility, small diameter, insulation, high spectral selectivity and reflectivity, mechanical compliance, electromagnetic interference immunity and so on[3,4]. The FBG sensors are rarely directly applied to monitor the displacement because of the intrinsic fragility of the optical fiber[5]. In the past studies, many works have been investigated concerning the feasibility of a displacement sensor based on an FBG. M. H. Yau and T. H.T chan et al.[6] have developed the curvature and inclination method for measuring vertical displacement by the FBG sensors. A beam loading test shows that the maximum discrepancies of vertical displacements using the curvature and inclination approaches are $0.14 \mathrm{~mm}$ and $0.41 \mathrm{~mm}$ respectively. M. Ciminello and S. Ameduri [7] have designed a dedicated device which constituted by a circular ring connected to the structure and laterally integrated with a FBG sensor for extending the FBG measurement range. Fangfang Liu and Yetai Fei et al.[8] have proposed and demonstrated a novel scheme which is composed of a double-FBG sensing structure and stainless steel tube for micro/nano displacement measurement. Hong-Hu Zhu and Jian-Hua Yin et al.[9] have used a FBG sensings adhered on a cantilever beam to perform real-time monitoring of internal displacements of a laboratoroy-scale physical model. Metje et al. have developed a fiberglass rod instrumented with FBG sensors for measuring structural displacements. 
Most of the above researches provided displacement measurements by FBG sensors which were adhered on the substrate such as an aluminum ring, steel tube and cantilever beam to avoid the intrinsic fragility of optical fiber. It is possible, by means of bending or inclination, to extend the range of displacement measurement. However, most of the FBG sensors could be used only once because FBG sensors and the substrate could not recover.

In this work, we proposed a novel method for design and curving an FBG sensor using auxiliary flexible driving function of the shape memory alloy (SMA) to monitor the displacement. The SMA has been regarded as one of functional materials for smart structures due to two outstanding effects which are known as the shape memory effect(SME) and super elastic(SE). The plastic deformation is applied to the SMA, it will shrinks to its original shape when it is heated at a certain temperature, which is called SME. The characteristic of SMA was conceived to overcome this fragility of the optical fiber, assuring multiple applications of FBG sensors.

\section{FBG sensing principles}

When an FBG sensor is irradiated with broadband light, a unique center wavelength called Bragg wavelength will be reflected. The Bragg wavelength is expressed as [10]:

$$
\lambda_{B}=2 \mathrm{n} \Lambda
$$

Where $\lambda_{B}$ is the Bragg wavelength, $\Lambda$ is the period of Bragg grating and $\mathrm{n}$ is the average refractive index of the optic fiber. The Bragg wavelength shifts in response to the fiber refractive index (n) and the grating pitch $(\Lambda)$, which vary with the changes in strain and temperature. Thus the Bragg wavelength shift $\left(\Delta \lambda_{B}\right.$ can:

$$
\frac{\Delta \lambda_{B}}{\lambda_{B}}=\left\{1-\left(\frac{\mathrm{n}^{2}}{2}\right)\left[\mathrm{P}_{12}-v\left(\mathrm{P}_{11}+\mathrm{P}_{12}\right)\right]\right\} \varepsilon+\left[\alpha+\frac{(\mathrm{dn} / \mathrm{dT})}{n}\right] \Delta \mathrm{T}
$$

where $\varepsilon$ is the applied strain; $\Delta \mathrm{T}$ is the temperature change; PijarePockel's (piezo) coefficients of the stress-optic tensor; $v$ is Poisson's ratio, and $\alpha$ is the coefficient of thermal expansion of the fiber material. The temperature varies are not considered, the thermal term can be neglected and Eq. 2 is simplified as :

$$
\frac{\Delta \lambda_{B}}{\lambda_{B}}=P_{e} \varepsilon
$$

where $p_{e}$ is the elasto-optic coefficient, which is calculated by the strain term of Eq. 2. From Eq. 3, it is linear relation between the $\Delta \lambda_{B}$ and optic-fiber axial strain change under constant temperature.

\section{Numerical simulation}

The FBG sensor was stuck on the surface of S shape SMA which was consist of two symmetric semicircle structure as shown in Fig. 1. The length of the S shape SMA was set as L. The cross section was circle and the diameter was set as a.

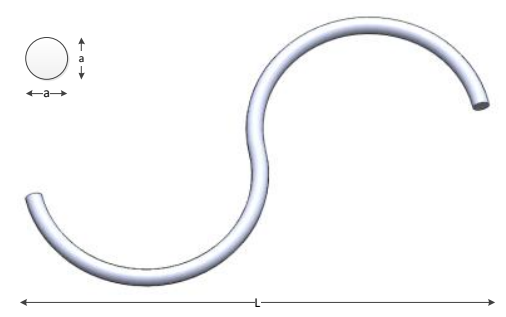

Fig. 1 S shape SMA 
The geometric model of S shape SMA established by SolidWorks was applied to ANASYS Workbench to simulate its strain. Material parameters of SMA for this simulation were set as following: elasticity modulus was $53 \mathrm{GPa}$, Poisson's ratio was 0.33 and density was $6450 \mathrm{~kg} / \mathrm{m} 3$. The clamping was fixed at the top node of S type SMA while the displacement load was applied at the opposite side in the horizontal direction. The simulations shown in Figs. 3 revealed that strain concentrates on the crest and trough of the symmetric semicircle structure. The FBG was supposed to be bonded on the crest or trough of the S type SMA.

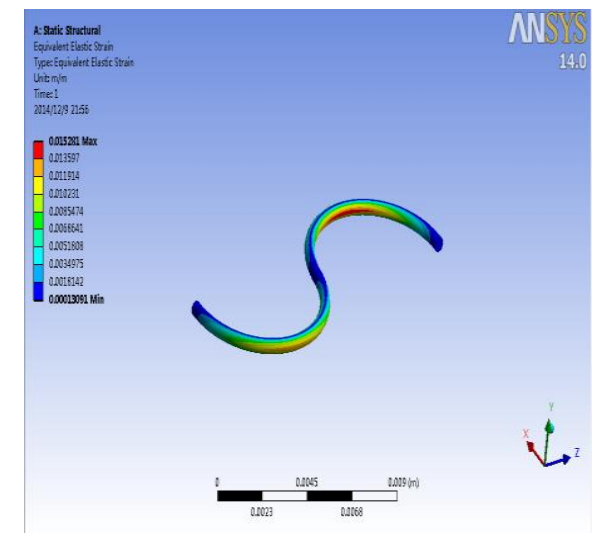

Fig. 2 Strain contours

The strain gradient was shown in Fig. 2 when the displacement was $2 \mathrm{~mm}$. The strain values of 6480 points were extracted according to the simulation result. Hence the FBG sensor with the length of $10 \mathrm{~mm}$, was divided averagely as 6480 sections. Each grating section which was corresponding with unique strain value was considered as an FBG. When $\lambda_{B}=1561.060 \mathrm{~nm}$, effective refractive index and its disturbance were 1.46 and 0.0001 respectively and $\Lambda=532 \mathrm{~nm}$, Spectrum of each grating section was calculated by Eq.(4) .

$$
R=\rho^{2}=\frac{\sinh ^{2}\left(\sqrt{(k L)^{2}-\left(\zeta^{+} L\right)^{2}}\right.}{-\frac{\zeta^{+^{2}}}{k^{2}}+\cosh ^{2} \sqrt{(k L)^{2}-\left(\zeta^{+} L\right)^{2}}}
$$

Here $\zeta=\frac{2 \pi}{\lambda} \overline{\Delta n}_{\text {eff }}, k=\frac{\pi}{\lambda} s \overline{\Delta n}_{\text {eff }}$ and coupling index $\mathrm{s}=1$. The corresponding reflection spectrum of curved FBG sensor was obtained by superimposing spectrums of 6480 grating sections. The spectral simulation result was shown in Fig. 3.

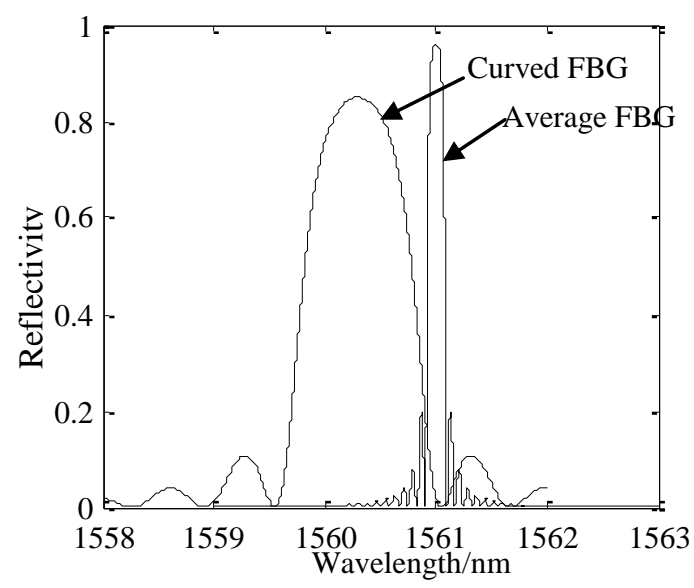

Fig. 3 Spectral simulations of curved FBG and average FBG 
When the curved FBG which was stuck on the surface of $S$ type SMA was stretched to $2 \mathrm{~mm}$, the bandwidth of spectrum was increased and the central wavelength was changed.

\section{Experiments}

SMA wire with a diameter of $0.5 \mathrm{~mm}$, which was set as $\mathrm{S}$ by molds at room temperature, was heated up to $450^{\circ} \mathrm{C}$ in the furnace and cooled two hours later. By above training SMA wire had the shape memory effect. The FBG sensor with an length of $20 \mathrm{~mm}$ was stuck on the surface of SMA wire which straightened by applying force. SMA wire Straightened can return to the S shape when exposed to the heat environment because of the shape memory effect. The left semicircle of S shape SMA with a radius of $3.18 \mathrm{~mm}$ was fixed to the testing frame and its right end was connected with optical platform which was driven by pole connected with micrometer. The optical platform moved by adjusting the micrometer, at the same time the FBG sensor stuck on the SMA wire was stretched. The spectrum changes of FBG sensor were obtained using the spectrograph. Fig. 4 illustrated the spectrum monitoring system used in this study.

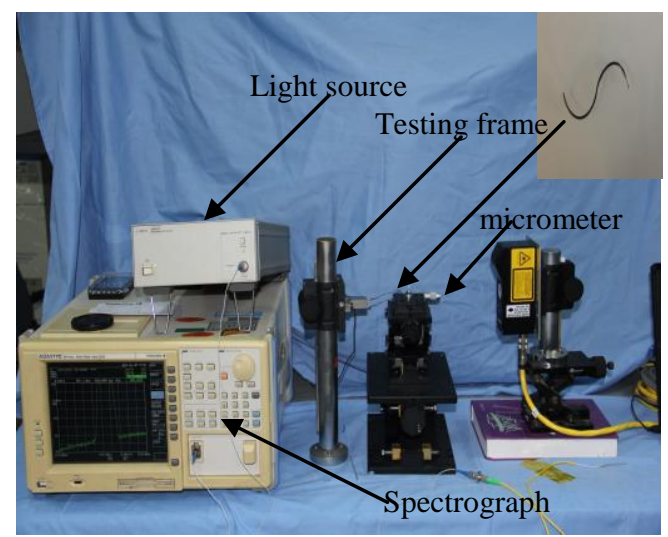

Fig. 4 Spectrum monitoring system

The $\mathrm{S}$ shape SMA was stretched from $1 \mathrm{~mm}$ to $6 \mathrm{~mm}$ by adjusting micrometer. A Spectrum was recorded when the scale of micrometer was changed at $0.5 \mathrm{~mm}$. The spectrums for different displacement were shown in Fig. 5.
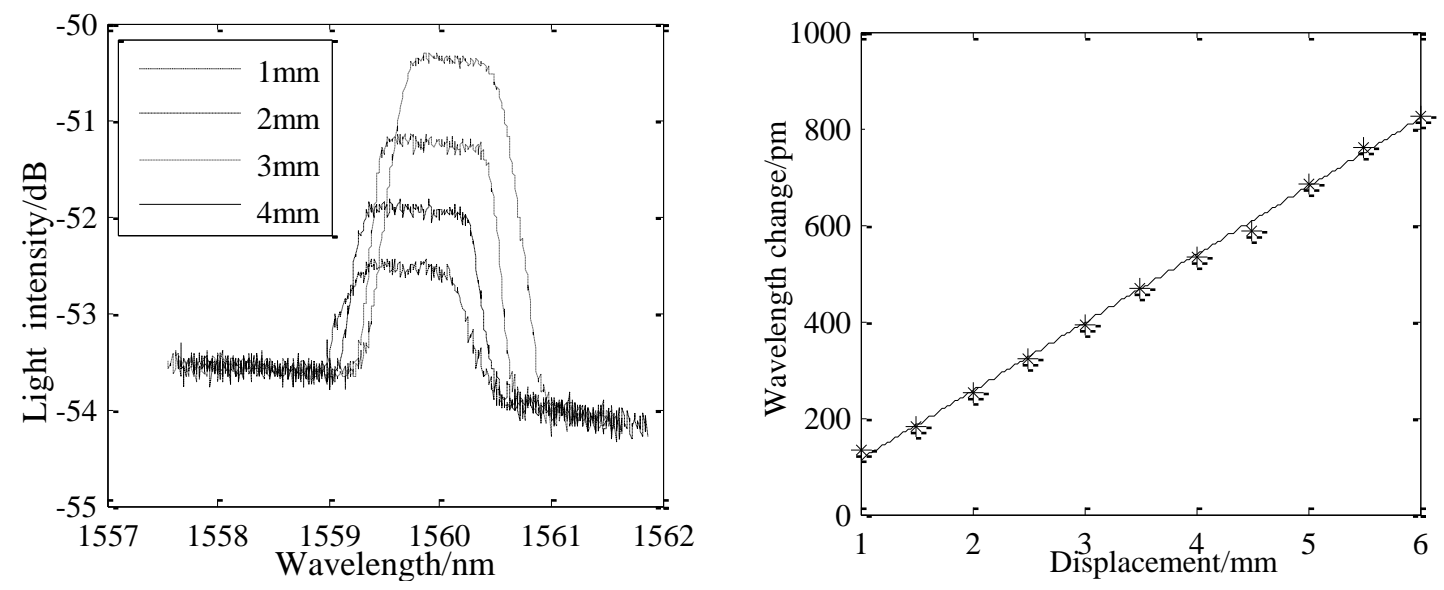

Fig. 5 Reflective spectrums Fig. 6 Relation of the displacement and wavelength change

\section{Results and discussion}

Fig. 5 showed the bandwidth of cured FBG was increased compared with average FBG and the light intensity of peak was increased with the increasing of displacement. The whole spectrum got red-shifted and the central wavelength was increased. The bandwidth of Curved FBG in inhomogeneous strain field, which could be seen from Fig. 2, was broadened because of its uniform 
of grating periods. The phenomenon was consistent with the spectral simulation. The grating was in tensional state in the process of stretching, hence the central wavelength was increased with the increasing of displacement. The intensity was increased because curved radian and strain gradients were decreased.

The relation between the central wavelength and displacement shown in Fig. 6 was linear and the wavelength shifted $141 \mathrm{pm}$ while displacement was changed $1 \mathrm{~mm}$. All of the above analysis revealed that curved FBG based on S shape SMA was used to be a displacement sensor.

\section{Conclusions}

A new displacement sensor was designed and fabricated by using the flexible bending property and shape memory effect of SMA. The FBG sensor stuck on the surface of straight SMA wire was curved slowly to an S shape along with SMA wire. When displacement loading was applied on S shape SMA, its Strain contours and curved FBG spectrum were simulated. Because the $S$ shape SMA was stretched the curved FBG sensor was in inhomogeneous strain field and the properties of reflective spectrum were changed. Its bandwidth, light intensity of peak and central wavelength were increased.

The central wavelength change was linear with displacement and the displacement sensitivity was $141 \mathrm{pm} / \mathrm{mm}$. The curved FBG can be used to monitor displacement within $6 \mathrm{~mm}$.

\section{Acknowledgments}

This research is supported by National Natural Science Foundation of China (No. 51405223)

\section{References}

[1] M.Y. Seung, J.H. Han and I. Lee: International Journal of Applied Electromagnetics and Mechanics, Vol.23 (2006) No.3, p.177-186.

[2] S. Takad, Y.Aoki and T. Ishikaw: Composite Structures, Vol.79 (2007) No.6, p.133-139.

[3] K.S.C. Kuang and W.J. Cantwell: Applied. Mechanics, Vol.56 (2003) No.4, p.493-513.

[4] S. Rapp, L.H. Kang and J.H. Han: Smart Material and Structures. Vol.18 (2009) No.6, p.25-37.

[5] H.C. Fan, Z. Zhang and X.J. Zhu: Mechanical\&Electrical Engineering Magazine. Vol.3(2009) No.6, p.41-43.

[6] M.H. Yau and T.H.T. Chan: Advances in Structural Engineering. Vol.16 (2013) No.1, p.165-176.

[7] M. Ciminello and S. Ameduri: Proc. IEEE MTT-S International Microwave and Optoelectronics Conference (Rio de Janeiro, Brazil, August 4-7, 2013). P.8-13.

[8] F.F. Liu, Y.T. Fei and H.J. Xia:Meas. Sci. Technol. Vol. 23 (2012) No.5, p.1-9.

[9] H.H. Zhu, J.H. Yin and L. Zhang: Advances in Structural Engineering. Vol.13 (2010) No.2, p.249-261.

[10] K.O. Hill, Y. Fuji and D.C. Johnson: Appl. Phys. Lett. Vol.32 (1978) No.10, p.647-649. 